\title{
Compilation and Analysis of Web-Based Orthopedic Personalized Predictive Tools: A Scoping Review
}

\author{
Patrick Curtin ${ }^{1}$, Alexandra Conway ${ }^{1}$, Liu Martin ${ }^{1}$, Eugenia Lin ${ }^{2}{ }^{\oplus}$, Prakash Jayakumar ${ }^{2}$ and \\ Eric Swart ${ }^{1, *}$ \\ 1 Department of Orthopedics, University of Massachusetts Medical Center, 55 N Lake Avenue, Worcester, \\ MA 01655, USA; Patrick.Curtin@umassmemorial.org (P.C.); Alexandra.Conway@umassmed.edu (A.C.); \\ Liu_Martin@branson.org (L.M.) \\ 2 Department of Surgery and Perioperative Care, University of Texas at Austin Dell Medical School, \\ 1601 Trinity Street, Austin, TX 78712, USA; Eugenia.Lin@austin.utexas.edu (E.L.); \\ Prakash.Jayakumar@austin.utexas.edu (P.J.) \\ * Correspondence: Eric.Swart@umassmemorial.org
}

Received: 9 October 2020; Accepted: 10 November 2020; Published: 12 November 2020

\begin{abstract}
Web-based personalized predictive tools in orthopedic surgery are becoming more widely available. Despite rising numbers of these tools, many orthopedic surgeons may not know what tools are available, how these tools were developed, and how they can be utilized. The aim of this scoping review is to compile and synthesize the profile of existing web-based orthopedic tools. We conducted two separate PubMed searches-one a broad search and the second a more targeted one involving high impact journals-with the aim of comprehensively identifying all existing tools. These articles were then screened for functional tool URLs, methods regarding the tool's creation, and general inputs and outputs required for the tool to function. We identified 57 articles, which yielded 31 unique web-based tools. These tools involved various orthopedic conditions (e.g., fractures, osteoarthritis, musculoskeletal neoplasias); interventions (e.g., fracture fixation, total joint arthroplasty); outcomes (e.g., mortality, clinical outcomes). This scoping review highlights the availability and utility of a vast array of web-based personalized predictive tools for orthopedic surgeons. Increased awareness and access to these tools may allow for better decision support, surgical planning, post-operative expectation management, and improved shared decision-making.
\end{abstract}

Keywords: web-based tools; orthopedics; predictive tools

\section{Introduction}

The ability to provide personalized predictions of clinical outcomes in the field of orthopedics is gaining interest [1-5]. Databases encompassing robust and accurate patient-level data [6-8], greater access to patient information via the electronic medical record [9], and the rise of advanced analytical capabilities, such as machine learning [10,11], provide the prospect of great strides in both our understanding of musculoskeletal problems and the outcomes of orthopedic interventions. Unlike simple risk calculations, web-based predictive tools analyze larger amounts of patient data and utilize algorithmic mathematical modeling and prediction analytics using advanced computing.

Despite the technological advances in predictive tools, many challenges exist in practical implementation of these solutions in clinical settings. Firstly, there is no common repository or standardized location to access personal predictive tools. These tools span various subspecialties within the field of orthopedics and other surgical specialties (e.g., capable of providing general risk calculations). As a result, where to find and identify tools appropriate for their clinical needs remains a barrier for orthopedic surgeons in practice. Secondly, once a tool has been identified for use, it can be 
difficult to discern how the tool was developed (i.e., what data inputs have been used to define the tool's algorithm) and how it has been assessed for technical feasibility and validated (i.e., the extent to which a tool can be used in a practice setting to fit a given need). Our overarching goal was to perform a scoping review to comprehensively map and organize the knowledge base around web-based personalized predictive tools in orthopedics.

Our primary objective was to map the current range of web-based predictive tools by type of data input, study characteristics and statistical methods used to develop the tool, type of data output, and the function of the tool. Our secondary objective was to qualitatively synthesize this data to generate a set of considerations for disseminating and implementing these tools in routine orthopedic practice. Our findings aim to provide orthopedic surgeons comprehensive insights into the range of available tools, researchers and technologists a premise to develop further innovative solutions in this field, and health systems a framework to integrate these tools to advance facets of orthopedic care.

\section{Methods}

We drafted a protocol a priori using the Preferred Reporting Items for Systematic Reviews and Meta-analysis Protocols (PRISMA-SCR), and members of our research team further refined the protocol through a collaborative process. Our protocol was registered through Open Science Framework on 24 September 2020 [12]. (To be included in this review, articles and studies needed to be in English, and include the uniform resource link (URL) for their web-based tool and/or sufficient information (references, tool name, researcher(s), institution, etc.) to find the tool. The development and validation studies mentioned in these articles needed to describe their patient population, intervention in the form of a personalized web-based predictive tool, methodology and description of their validation and development, outcomes provided by the web-based tool, and type of study. Articles referencing studies for which this information was missing were excluded. Exclusion criteria also included articles without URLs or sufficient information to identify the tool, article links with no access to full-text PDFs, and articles written in languages other than English. Articles about tools with no orthopedic relevance, such as a tool predicting cardiovascular disease risk in patients taking statins, were also excluded from this study. For the purposes of this review, prospective cohort studies, retrospective cohort studies, and meta-analyses were included, while case reports were excluded.

To identify web-based tools that fit the above inclusion criteria, a comprehensive search of the bibliographic electronic database PubMed (NLM) was conducted using specific search terms and with no time-period restriction. Search terms were initially drafted by an experienced orthopedic surgeon familiar with web-based orthopedic tools, and further refined through discussion among the research team. Search results were then screened for the above inclusion criteria and included only if all criteria were met. To ensure the comprehensive capture of any additional web-tools, a second more targeted search was performed, focusing on a set of high-impact orthopedic journals within each subspecialty. Targeted subspecialties are listed in Table 1. The list of high-impact orthopedic journals was created by an experienced orthopedic surgeon and the research team. These journals are listed in Appendix A. Duplicate articles were removed for both searches. After identifying all tools, missing original development and validation articles were found and included for any tools that lacked them in the initial searches. The final search strategy with both primary and secondary searches is recorded in Appendix B. 
Table 1. Targeted Orthopedic Subspecialties Used in Secondary Search.

\begin{tabular}{c}
\hline Joint Replacement \\
Research \\
Trauma \\
Sports \\
Hand and Upper Extremity \\
Shoulder and Elbow \\
Foot and Ankle \\
Spine \\
Pediatric \\
\hline
\end{tabular}

Screening of the identified articles was performed by all members of the research team. For articles that passed preliminary screening, we downloaded the full-text versions of the studies, mostly as portable document files (PDFs). We subsequently extracted and recorded relevant data using an electronic data collection sheet. For each tool we extracted the following parameters: tool name, functional URL link, user input data, tool outputs, type of study used for tool creation and/or validation, number of patients involved in those studies, and statistical methods used for creation of tool. User inputs were categorized into demographic, clinical, and patient reported data. Clinical input data were defined to include medical, biometric, and radiologic findings. Categorical examples of user input data are listed in Table 2. Tools were then grouped by output category, examples for which are listed in Table 3.

Table 2. Types of User Input Data.

\begin{tabular}{cc}
\hline Category & Examples \\
\hline Demographic & Age, Sex, Body Mass Index (BMI) \\
\hline Clinical & Medical History, Injury Characteristics, Procedure Characteristics \\
\hline Patient Reported & $\begin{array}{c}\text { Social Information (e.g., housing, sexual activity, recreational activities, } \\
\text { etc.), Smoking Status, Current Alcohol Use, Recent Fall History }\end{array}$ \\
\hline
\end{tabular}

Table 3. Types of Tool Output Data.

\begin{tabular}{|c|c|}
\hline Category & Examples \\
\hline Fracture Prediction & Loss of Position, Risk of Fracture \\
\hline Mortality Prediction & Survival Rates \\
\hline Clinical Events Prediction & $\begin{array}{c}\text { Surgical Complications, Post-Operative Pain, Readmission } \\
\text { Rates, Treatment Options }\end{array}$ \\
\hline Processes Prediction & Length of Stay, Discharge Disposition \\
\hline
\end{tabular}

\section{Results}

We identified 358 total records through our search strategy, 57 of which matched our inclusion criteria. From those 57 articles, 31 unique tools were identified, analyzed, and included for this scoping review (Table 4). There was a higher number of articles identified compared to tools because some articles were reviews of multiple tools or re-validation studies of already identified tools. Useable links for each tool are listed in Appendix C. 
Table 4. Tools Categorized by Output.

\begin{tabular}{|c|c|c|c|c|c|c|c|}
\hline Tool & $\begin{array}{l}\text { Journal and (Year) of } \\
\text { Publication [Ref] }\end{array}$ & $\begin{array}{l}\text { Tool Development } \\
\text { Study Type and Size }\end{array}$ & $\begin{array}{l}\text { Demographic } \\
\text { Input }\end{array}$ & Clinical Input & $\begin{array}{l}\text { Patient Reported } \\
\text { Input }\end{array}$ & Tool Output & $\begin{array}{c}\text { Statistical } \\
\text { Methods Used in } \\
\text { Tool } \\
\text { Development } \\
\end{array}$ \\
\hline $\begin{array}{l}\text { Edinburgh Wrist } \\
\text { Calculator }\end{array}$ & $\begin{array}{l}\text { Journal of Orthopaedic Trauma } \\
\text { (2018) [13,14], The Journal of Bone } \\
\text { and Joint Surgery (2006) [15] }\end{array}$ & $\begin{array}{l}\text { Prospective Cohort } \\
4000 \text { distal radius } \\
\text { fractures }\end{array}$ & Age & $\begin{array}{l}\text { Ulnar Variance, Dorsal Comminution } \\
\text { Present, Physical Dependence }\end{array}$ & & $\begin{array}{l}\text { Fracture prediction: } \\
\text { Loss of position }\end{array}$ & $\begin{array}{l}\text { Univariate and } \\
\text { Multiple Logistic } \\
\text { Regression }\end{array}$ \\
\hline $\begin{array}{l}\text { CAROC } \\
\text { (Canadian Risk for } \\
\text { Osteoporosis } \\
\text { Calculator) }\end{array}$ & $\begin{array}{l}\text { Journal of Clinical Densitometry } \\
\text { (2017) [16], (2010) [17], (2007) [18] }\end{array}$ & $\begin{array}{l}\text { Retrospective Cohort } \\
39,603 \text { patients }\end{array}$ & Age, Sex & $\begin{array}{l}\text { Femoral Neck T-Score, Fragility } \\
\text { Fracture after age } 40, \text { Recent prolonged } \\
\text { glucocorticoid use }\end{array}$ & & $\begin{array}{l}\text { Fracture prediction: } \\
\text { Risk categorization }\end{array}$ & $\begin{array}{l}\text { Kaplan-Maier } \\
\text { Method }\end{array}$ \\
\hline FRAX & $\begin{array}{l}\text { Journal of Clinical Densitometry } \\
\text { (2017) [19], Turkish Journal of } \\
\text { Urology (2019) [20] }\end{array}$ & $\begin{array}{l}\text { Systematic Review } \\
290,000 \text { patients }\end{array}$ & $\begin{array}{l}\text { Age, Sex, Height, } \\
\text { Weight }\end{array}$ & $\begin{array}{l}\text { Previous Fracture, Glucocorticoids, } \\
\text { Rheumatoid arthritis, Secondary } \\
\text { Osteoporosis, Femoral neck BMD }\end{array}$ & $\begin{array}{l}\text { Smoking status, } \\
\text { Alcohol use, } \\
\text { Parental history of } \\
\text { hip fracture }\end{array}$ & $\begin{array}{l}\text { Fracture prediction: } \\
\text { 10-year risk }\end{array}$ & $\begin{array}{l}\text { Multiple Logistic } \\
\text { Regression }\end{array}$ \\
\hline $\begin{array}{l}\text { FRS (Fracture Risk } \\
\text { Scale) }\end{array}$ & $\begin{array}{c}\text { BioMed Central (BMC) Geriatrics } \\
\text { (2018) [21], British Medical Journal } \\
\text { (BMJ) Open (2017) [22] }\end{array}$ & $\begin{array}{l}\text { Retrospective Cohort } \\
29,848 \text { patients }\end{array}$ & Age, BMI & $\begin{array}{l}\text { Wandering frequency, Walking in } \\
\text { corridor, Transfer status, Cognitive } \\
\text { performance scale, hip fracture history }\end{array}$ & Fall history & $\begin{array}{l}\text { Fracture prediction: } \\
\text { 1-year hip fracture risk }\end{array}$ & $\begin{array}{l}\text { Decision Tree and } \\
\text { Logistic } \\
\text { Regression }\end{array}$ \\
\hline Garvan & $\begin{array}{l}\text { Journal of Clinical Densitometry } \\
\text { (2017) [19], Osteoporosis } \\
\text { International (2008) [23] }\end{array}$ & $\begin{array}{l}\text { Prospective Cohort } \\
2216 \text { patients }\end{array}$ & Age, Sex & $\begin{array}{l}\text { Fractures and fall history, T-scores, } \\
\text { actual BMD }\end{array}$ & & $\begin{array}{l}\text { Fracture prediction: 5- } \\
\text { and 10-year risk }\end{array}$ & $\begin{array}{l}\text { Cox's Proportional } \\
\text { Hazards Analysis }\end{array}$ \\
\hline QFracture & $\begin{array}{c}\text { Journal of Clinical Densitometry } \\
\text { (2017) [16,19], The British Medical } \\
\text { Journal (Clinical research ed.) } \\
\text { (2012) [24] }\end{array}$ & $\begin{array}{l}\text { Prospective Cohort } \\
4,726,046 \text { patients }\end{array}$ & $\begin{array}{l}\text { Age, Sex, BMI, } \\
\text { Ethnicity }\end{array}$ & $\begin{array}{c}\text { Diabetes, osteoporotic fracture history, } \\
\text { Dementia, Cancer, Asthma or COPD, } \\
\text { Cardiovascular disease, liver disease, } \\
\text { kidney disease, Parkinson's, } \\
\text { Rheumatoid arthritis, SLE, GI } \\
\text { malabsorption, Endocrine problems, } \\
\text { Epilepsy, Hormone Therapy, use of } \\
\text { anticonvulsants/ } \\
\text { antidepressants/corticosteroids/estrogen }\end{array}$ & $\begin{array}{l}\text { Smoking status, } \\
\text { Alcohol use, Fall } \\
\text { history, Parental } \\
\text { history of hip } \\
\text { fracture/osteoporosis, } \\
\text { Residence }\end{array}$ & $\begin{array}{l}\text { Fracture prediction: } \\
\text { 10-year risk }\end{array}$ & $\begin{array}{l}\text { Multivariate Final } \\
\text { Cox Regression }\end{array}$ \\
\hline $\begin{array}{c}\text { NHFS } \\
\text { (Nottingham Hip } \\
\text { Fracture Score) }\end{array}$ & $\begin{array}{l}\text { British Journal of Anaesthesia (2008) } \\
\text { [25], The Bone \& Joint Journal } \\
\text { (2015) [26], Injury (2015) [27] }\end{array}$ & $\begin{array}{l}\text { Prospective Cohort } \\
4967 \text { patients }\end{array}$ & Age, Sex & $\begin{array}{c}\text { AMTS, } \mathrm{Hb} \text { on admission, } \\
\text { Comorbidities, Active malignancy } \\
\text { history }\end{array}$ & Residence & $\begin{array}{l}\text { Fracture prediction: } \\
\text { NHFS Score } \\
\text { Mortality prediction }\end{array}$ & $\begin{array}{l}\text { Forward } \\
\text { Univariate and } \\
\text { Multivariate } \\
\text { Logistic } \\
\text { Regression }\end{array}$ \\
\hline
\end{tabular}


Table 4. Cont.

\begin{tabular}{|c|c|c|c|c|c|c|c|}
\hline Tool & $\begin{array}{l}\text { Journal and (Year) of } \\
\text { Publication [Ref] }\end{array}$ & $\begin{array}{l}\text { Tool Development } \\
\text { Study Type and Size }\end{array}$ & $\begin{array}{c}\text { Demographic } \\
\text { Input }\end{array}$ & Clinical Input & $\begin{array}{l}\text { Patient Reported } \\
\text { Input }\end{array}$ & Tool Output & $\begin{array}{c}\text { Statistical } \\
\text { Methods Used in } \\
\text { Tool } \\
\text { Development }\end{array}$ \\
\hline $\begin{array}{c}\text { ACS NSQIP } \\
\text { Surgical Risk } \\
\text { Calculator }\end{array}$ & $\begin{array}{l}\text { Journal of Neurosurgery. Spine } \\
\text { (2017) [28], The Journal of } \\
\text { Arthroplasty (2018) [29], Spine } \\
\text { (2020) [30], Clinical Orthopaedics } \\
\text { and Related Research (2016) [31], } \\
\text { The Journal of Arthroplasty (2015) } \\
\text { [32], Journal of the American College } \\
\text { of Surgeons (2013) [33] }\end{array}$ & $\begin{array}{l}\text { Retrospective Cohort } \\
1,414,006 \text { patients }\end{array}$ & $\begin{array}{l}\text { Age, Sex, Height, } \\
\text { Weight }\end{array}$ & $\begin{array}{c}\text { Procedure, Functional Status, } \\
\text { Emergency Case, ASA Class, Steroid } \\
\text { use, Ascites, Systemic Sepsis, Ventilator } \\
\text { Dependent, Disseminated Cancer, } \\
\text { Diabetes, Hypertension, CHF, Dyspnea, } \\
\text { History of Severe COPD, Dialysis, } \\
\text { Acute Renal Failure }\end{array}$ & Smoking status & $\begin{array}{l}\text { Mortality prediction } \\
\text { Clinical events } \\
\text { prediction: Adverse } \\
\text { events } \\
\text { Processes prediction: } \\
\text { Length of stay }\end{array}$ & $\begin{array}{l}\text { Random Intercept } \\
\text { and Fixed Slope } \\
\text { Hierarchical } \\
\text { Models }\end{array}$ \\
\hline $\begin{array}{c}\text { E-PASS } \\
\text { (Estimation of } \\
\text { Physiologic Ability } \\
\text { and Surgical } \\
\text { Stress) Surgery } \\
\text { Risk Calculator }\end{array}$ & $\begin{array}{c}\text { Journal of Bone and Mineral } \\
\text { Research [34], Injury (2015) [27], } \\
\text { Surgery Today (1999) [35], Surgery } \\
\text { (2004) [36] }\end{array}$ & $\begin{array}{l}\text { Retrospective Cohort } \\
3981 \text { patients }\end{array}$ & Age, Weight & $\begin{array}{l}\text { Cardiac arrhythmia, Pulmonary vital } \\
\text { capacity, FEV1, Diabetes, Blood loss, } \\
\text { OR time, Extent of skin incision at } \\
\text { surgery, Heart failure, ECOG } \\
\text { performance status, ASA class }\end{array}$ & & $\begin{array}{l}\text { Clinical event } \\
\text { prediction: } \\
\text { Preoperative risk } \\
\text { score, Surgical stress } \\
\text { score, Comprehensive } \\
\text { risk score }\end{array}$ & $\begin{array}{l}\text { Multiple Logistic } \\
\text { Regression }\end{array}$ \\
\hline $\begin{array}{l}\text { STTGMA (Score } \\
\text { for Trauma Triage } \\
\text { in Geriatric and } \\
\text { Middle Aged } \\
\text { Patients) }\end{array}$ & $\begin{array}{l}\text { The Journal of the American } \\
\text { Academy of Orthopaedic Surgeons } \\
\text { (2020) [37], Bulletin of the Hospital } \\
\text { for Joint Disease (2016) [38] }\end{array}$ & $\begin{array}{l}\text { Retrospective Cohort } \\
138,096 \text { patients }\end{array}$ & Age & $\begin{array}{l}\text { Injury mechanism, Glasgow Coma } \\
\text { Scale (GCS), Abbreviated Injury Scale } \\
\text { (AIS), Head/Neck, Chest, Extremity, } \\
\text { Charlson Comorbidity Index (CCI) }\end{array}$ & & $\begin{array}{l}\text { Mortality prediction: } \\
\text { STIGMA Score }\end{array}$ & $\begin{array}{l}\text { Logistic } \\
\text { Regression } \\
\text { Analysis }\end{array}$ \\
\hline $\begin{array}{l}\text { CCI (Charlson } \\
\text { Comorbidity } \\
\text { Index) }\end{array}$ & $\begin{array}{c}\text { Journal of Neurosurgery. Spine } \\
\text { (2017) [28], Clinical Orthopaedics } \\
\text { and Related Research (2014) [39], } \\
\text { Injury (2015) [27], Journal of } \\
\text { Orthopaedic Research (2020) [40], } \\
\text { Journal of Chronic Diseases (1987) } \\
\text { [41] }\end{array}$ & $\begin{array}{l}\text { Prospective Cohort } \\
1244 \text { patients }\end{array}$ & Age & $\begin{array}{l}\text { Myocardial infraction, CHF, Peripheral } \\
\text { vascular disease, CVA or TIA, } \\
\text { Dementia, COPD, Connective tissue } \\
\text { disease, Peptic ulcer disease, Liver } \\
\text { disease, Diabetes Mellitus, Hemiplegia, } \\
\text { Moderate to severe CKD, Solid tumor, } \\
\text { Leukemia, Lymphoma, AIDS }\end{array}$ & & $\begin{array}{l}\text { Mortality prediction: } \\
\text { CCI, 10-year survival }\end{array}$ & $\begin{array}{l}\text { Kaplan-Maier } \\
\text { Method }\end{array}$ \\
\hline P-POSSUM & $\begin{array}{l}\text { The British Journal of Surgery (1991) } \\
\text { [42], (1998) [43], Injury (2015) [27] }\end{array}$ & $\begin{array}{l}\text { Prospective Cohort } \\
1440 \text { patients }\end{array}$ & Age & $\begin{array}{c}\text { Cardiac status, Respiratory status, } \\
\text { ECG, Systolic BP, Pulse, Hemoglobin, } \\
\text { WBC, Urea, Sodium, Potassium, GCS, } \\
\text { Operation type, Number of procedures, } \\
\text { Operative Blood Loss, Peritoneal } \\
\text { Contamination, Malignancy Status, } \\
\text { CEPOD }\end{array}$ & & $\begin{array}{l}\text { Mortality prediction } \\
\text { (for esophagogastric } \\
\text { surgery) } \\
\text { Clinical event } \\
\text { prediction: Physiology } \\
\text { score, Operative } \\
\text { severity score, } \\
\text { Morbidity }\end{array}$ & $\begin{array}{c}\text { Multiple Logistic } \\
\text { Regression }\end{array}$ \\
\hline
\end{tabular}


Table 4. Cont.

\begin{tabular}{|c|c|c|c|c|c|c|c|}
\hline Tool & $\begin{array}{l}\text { Journal and (Year) of } \\
\text { Publication [Ref] }\end{array}$ & $\begin{array}{l}\text { Tool Development } \\
\text { Study Type and Size }\end{array}$ & $\begin{array}{c}\text { Demographic } \\
\text { Input }\end{array}$ & Clinical Input & $\begin{array}{l}\text { Patient Reported } \\
\text { Input }\end{array}$ & Tool Output & $\begin{array}{c}\text { Statistical } \\
\text { Methods Used in } \\
\text { Tool } \\
\text { Development } \\
\end{array}$ \\
\hline LACE+ & $\begin{array}{l}\text { Journal of Orthopaedic Research } \\
\text { (2020) [40], Canadian Medical } \\
\text { Association Journal (2010) [44], } \\
\text { Open Medicine (2012) [45] }\end{array}$ & $\begin{array}{l}\text { Prospective Cohort } \\
500,000 \text { patients }\end{array}$ & & $\begin{array}{c}\text { Length of stay, Acuity of admission, } \\
\text { Comorbidities, Emergency department } \\
\text { visits }\end{array}$ & & $\begin{array}{l}\text { Mortality prediction: } \\
\text { LACE+ score } \\
\text { Clinical event } \\
\text { prediction: } \\
\text { Readmission risk }\end{array}$ & $\begin{array}{c}\text { Split-Sample } \\
\text { Design, Fractional } \\
\text { Polynomial } \\
\text { Functions, } \\
\text { Multiple Logistic } \\
\text { Regression }\end{array}$ \\
\hline $\begin{array}{l}\text { Chondrosarcoma } \\
\text { Five-Year Survival } \\
\text { Machine Learning } \\
\text { Algorithm }\end{array}$ & $\begin{array}{l}\text { Clinical Orthopaedics and Related } \\
\text { Research (2018) [46] }\end{array}$ & $\begin{array}{l}\text { Retrospective Cohort } \\
1554 \text { patients }\end{array}$ & Age, Sex & $\begin{array}{l}\text { Histology, Size, Extension, Grade, } \\
\text { Location }\end{array}$ & & $\begin{array}{l}\text { Mortality prediction: } \\
5 \text {-year survival }\end{array}$ & $\begin{array}{c}\text { Nonparametric } \\
\text { Missforest Method, } \\
\text { Boosted Decision } \\
\text { Tree, Support } \\
\text { Vector Machine, } \\
\text { Bayes Point } \\
\text { Machine, Neural } \\
\text { Network Models, } \\
\text { 10-Fold } \\
\text { Crossvalidation }\end{array}$ \\
\hline $\begin{array}{l}\text { Extremity } \\
\text { Metastatic Disease } \\
\text { Survival } \\
\text { Prediction } \\
\text { Machine Learning } \\
\text { Algorithm }\end{array}$ & $\begin{array}{l}\text { Clinical Orthopaedics and Related } \\
\text { Research (2020) [47] }\end{array}$ & $\begin{array}{l}\text { Retrospective Cohort } \\
1090 \text { patients }\end{array}$ & Age & $\begin{array}{l}\text { Primary Tumor Histology, Visceral } \\
\text { Metastasis, Brain Metastasis, Previous } \\
\text { Systemic Therapy, Hemoglobin, } \\
\text { Platelet, Absolute Lymphocyte, } \\
\text { Absolute Neutrophil, Creatinine, White } \\
\text { Blood Cell, Albumin, Alkaline } \\
\text { Phosphatase, Sodium, Calcium }\end{array}$ & & $\begin{array}{l}\text { Mortality prediction: } \\
\text { 90-day and 1-year } \\
\text { survival }\end{array}$ & $\begin{array}{c}\text { Missforest } \\
\text { Methods, Random } \\
\text { Forest Algorithms, } \\
\text { Stochastic } \\
\text { Gradient Boosting, } \\
\text { Random Forest, } \\
\text { Support Vector } \\
\text { Machine, Neural } \\
\text { Network, and } \\
\text { Penalized Logistic } \\
\text { Regression }\end{array}$ \\
\hline PathFX & $\begin{array}{l}\text { Clinical Orthopaedics and Related } \\
\text { Research (2017) [48], BioMed } \\
\text { Central (BMC) Cancer (2015) [49] }\end{array}$ & $\begin{array}{l}\text { Retrospective Cohort } \\
1291 \text { patients }\end{array}$ & Age, Sex & $\begin{array}{l}\text { Oncologic Diagnosis, Pathologic } \\
\text { Fracture, ECOG Performance Status, } \\
\text { Hemoglobin concentration, Absolute } \\
\text { lymphocyte count, Skeletal Metastases, } \\
\text { Organ Metastases, Lymph Node } \\
\text { Metastases, Physician's Estimate of } \\
\text { Survival, Skeletal Region }\end{array}$ & & $\begin{array}{l}\text { Mortality prediction: } \\
\text { Survival likelihood } \\
\text { after treatment at } \\
\text { multiple time intervals } \\
\text { Clinical event } \\
\text { prediction: Potential } \\
\text { treatments }\end{array}$ & $\begin{array}{l}\text { Bayesian Belief } \\
\text { Networks }\end{array}$ \\
\hline
\end{tabular}


Table 4. Cont.

\begin{tabular}{|c|c|c|c|c|c|c|c|}
\hline Tool & $\begin{array}{l}\text { Journal and (Year) of } \\
\text { Publication [Ref] }\end{array}$ & $\begin{array}{l}\text { Tool Development } \\
\text { Study Type and Size }\end{array}$ & $\begin{array}{l}\text { Demographic } \\
\text { Input }\end{array}$ & Clinical Input & $\begin{array}{l}\text { Patient Reported } \\
\text { Input }\end{array}$ & Tool Output & $\begin{array}{c}\text { Statistical } \\
\text { Methods Used in } \\
\text { Tool } \\
\text { Development }\end{array}$ \\
\hline $\begin{array}{l}\text { Incidental } \\
\text { Durotomy } \\
\text { Calculator }\end{array}$ & $\begin{array}{l}\text { Journal of Neurosurgery. Spine } \\
\text { (2020) [50] }\end{array}$ & $\begin{array}{l}\text { Retrospective Review } \\
1279 \text { patients }\end{array}$ & Age & $\begin{array}{l}\text { Revision Procedure, Procedure start } \\
\text { after } 4 \mathrm{pm} \text {, Surgery duration }\end{array}$ & & $\begin{array}{l}\text { Clinical event } \\
\text { prediction: Incidental } \\
\text { durotomy likelihood }\end{array}$ & $\begin{array}{c}\text { Natural language } \\
\text { processing (NLP), } \\
\text { Multiple Logistic } \\
\text { Regression, } \\
\text { Bootstrapping }\end{array}$ \\
\hline $\begin{array}{l}\text { Spinal RAT (Risk } \\
\text { Assessment Tool) }\end{array}$ & $\begin{array}{l}\text { Journal of Neurosurgery. Spine } \\
\text { (2017) [28] }\end{array}$ & $\begin{array}{c}\text { Prospective and } \\
\text { Retrospective Cohort } \\
279,391 \text { patients }\end{array}$ & Age, Sex & $\begin{array}{l}\text { Spinal Area, Pre-op diagnosis, Use of } \\
\text { BMP, Fusion, Surgery level, } \\
\text { Instrumentation, Pulmonary } \\
\text { dysfunction, Neurologic dysfunction, } \\
\text { Hypercholesterolemia, Hypertension, } \\
\text { Cardiac dysfunction, Diabetes mellitus, } \\
\text { Systemic malignancy, } \\
\text { Gastroesophageal dysfunction, } \\
\text { Psychiatric disorder, Substance abuse }\end{array}$ & Smoking status & $\begin{array}{l}\text { Clinical event } \\
\text { prediction: Surgical } \\
\text { complications risk (\%), } \\
\text { risk classification (low, } \\
\text { medium, high) }\end{array}$ & $\begin{array}{c}\text { Logistic } \\
\text { Regression with } \\
\text { Main Effects, } 2 \text { and } \\
3 \text { Factor } \\
\text { Interactions }\end{array}$ \\
\hline SpineSage & The Spine Journal (2014) [51] & $\begin{array}{l}\text { Prospective Cohort } \\
1532 \text { patients }\end{array}$ & Age, Gender, BMI & $\begin{array}{l}\text { Primary Diagnosis, Level of Surgery, } \\
\text { Surgical Approach, Cerebrovascular } \\
\text { Disease, Chronic Obstructive } \\
\text { Pulmonary Disease, Asthma, } \\
\text { Hypertension, Rheumatoid Arthritis, } \\
\text { Renal Conditions, Pre-existing } \\
\text { Neoplasm, Syncope or Seizure, } \\
\text { Anemia, Bleeding disorder, Diabetes, } \\
\text { CHF, Revision surgery, Previous spinal } \\
\text { surgery, Previous cardiac complications }\end{array}$ & & $\begin{array}{l}\text { Clinical event } \\
\text { prediction: } \\
\text { Complications risk }\end{array}$ & $\begin{array}{l}\text { Multivariate } \\
\text { Analysis }\end{array}$ \\
\hline $\begin{array}{l}\text { Back Treatment } \\
\text { Outcomes } \\
\text { Calculator }\end{array}$ & Spine (2002) [52], (2018) [53] & $\begin{array}{l}\text { Prospective Cohort } \\
289 \text { patients }\end{array}$ & $\begin{array}{l}\text { Age, Sex, Height, } \\
\text { Weight }\end{array}$ & $\begin{array}{c}\text { Condition, Symptoms, Episode, } \\
\text { Hypertension, Physical therapy, } \\
\text { Depression }\end{array}$ & $\begin{array}{c}\text { Smoking status, } \\
\text { Activities, Work } \\
\text { status, Worker's } \\
\text { compensation, } \\
\text { Education, } \\
\text { Expectation, Sleep, } \\
\text { Sex life }\end{array}$ & $\begin{array}{l}\text { Clinical event } \\
\text { prediction: Treatment } \\
\text { risks, Outcomes with } \\
\text { or without surgery } \\
\text { (physical functioning, } \\
\text { pain, sleep, sex life, } \\
\text { satisfaction with } \\
\text { symptoms) }\end{array}$ & $\begin{array}{l}\text { Multivariate } \\
\text { Analysis }\end{array}$ \\
\hline
\end{tabular}


Table 4. Cont.

\begin{tabular}{|c|c|c|c|c|c|c|c|}
\hline Tool & $\begin{array}{l}\text { Journal and (Year) of } \\
\text { Publication [Ref] }\end{array}$ & $\begin{array}{l}\text { Tool Development } \\
\text { Study Type and Size }\end{array}$ & $\begin{array}{l}\text { Demographic } \\
\text { Input }\end{array}$ & Clinical Input & $\begin{array}{l}\text { Patient Reported } \\
\text { Input }\end{array}$ & Tool Output & $\begin{array}{c}\text { Statistical } \\
\text { Methods Used in } \\
\text { Tool } \\
\text { Development }\end{array}$ \\
\hline $\begin{array}{c}\text { OaraScore } \\
\text { (Outpatient } \\
\text { Arthroplasty Risk } \\
\text { Assessment Score) }\end{array}$ & $\begin{array}{l}\text { The Journal of Arthroplasty (2018) } \\
\text { [54], (2017) [55] }\end{array}$ & $\begin{array}{l}\text { Retrospective Review } \\
1120 \text { patients }\end{array}$ & BMI & $\begin{array}{l}\text { Chronic narcotic use, Chronic pain } \\
\text { control difficulty, Chronic } \\
\text { benzodiazepine use, Severe } \\
\text { deconditioning; Additional inputs by } \\
\text { topic }\end{array}$ & $\begin{array}{l}\text { Lack of home } \\
\text { support }\end{array}$ & $\begin{array}{l}\text { Clinical event } \\
\text { prediction: Outpatient } \\
\text { surgery risk, } \\
\text { Assessment score }\end{array}$ & $\begin{array}{l}\text { Multivariate } \\
\text { Analysis }\end{array}$ \\
\hline $\begin{array}{l}\text { Arthroplasty Size } \\
\text { Predictor }\end{array}$ & $\begin{array}{l}\text { The Journal of Arthroplasty (2019) } \\
\text { [56], (2017) [57] }\end{array}$ & $\begin{array}{l}\text { Retrospective Cohort } \\
3491 \text { primary TKAs }\end{array}$ & $\begin{array}{l}\text { Sex, Height, } \\
\text { Weight }\end{array}$ & Manufacturer, Model & & $\begin{array}{l}\text { Clinical prediction: } \\
\text { Predicted sizes }\end{array}$ & $\begin{array}{c}\text { Multivariate } \\
\text { Linear Regression }\end{array}$ \\
\hline $\begin{array}{l}\text { 90-Day } \\
\text { Readmissions Risk } \\
\text { Calculator }\end{array}$ & $\begin{array}{l}\text { The Journal of Bone and Joint } \\
\text { Surgery (2019) [58] }\end{array}$ & $\begin{array}{c}\text { Retrospective Cohort } \\
\text { 10,155 THAs and } \\
\text { TKAs }\end{array}$ & Age & $\begin{array}{l}\text { Joint, ASA, Duration of Surgery, } \\
\text { Hemoglobin Level Postoperative, } \\
\text { Cardiac Arrhythmia, CHF, Chronic } \\
\text { Pulmonary Disease, Diabetes } \\
\text { Complicated, Hypertension, } \\
\text { Lymphoma, Neurologic Disease, } \\
\text { Peripheral Vascular Disease, } \\
\text { Pulmonary Circulation Disease, Renal } \\
\text { Failure, Depression, Substance abuse }\end{array}$ & $\begin{array}{l}\text { Smoking Status, } \\
\text { Alcohol use }\end{array}$ & $\begin{array}{l}\text { Clinical event } \\
\text { prediction: } 90 \text {-day } \\
\text { readmission risk }\end{array}$ & $\begin{array}{l}\text { Multiple Logistic } \\
\text { Regression }\end{array}$ \\
\hline $\begin{array}{l}\text { QUALITOUCH } \\
\text { Outcome } \\
\text { Calculator }\end{array}$ & $\begin{array}{l}\text { Journal of Telemedicine and Telecare } \\
\text { (2014) [59] }\end{array}$ & $\begin{array}{l}\text { Prospective Cohort } \\
483 \text { patients }\end{array}$ & $\begin{array}{l}\text { Age, Sex, Height, } \\
\text { Weight }\end{array}$ & $\begin{array}{c}\text { Type of surgery, Hip replacement, Knee } \\
\text { replacement, Spine surgery, } \\
\text { Hypertension, Heart disease, Stroke, } \\
\text { Depression, Diabetes, Cancer, } \\
\text { Lung/Kidney/GI disease, Anemia, } \\
\text { Substance abuse }\end{array}$ & $\begin{array}{c}\text { Pain during } \\
\text { activities, } \\
\text { Difficulty in } \\
\text { activities/movements, } \\
\text { Chronic pain, Back } \\
\text { pain }\end{array}$ & $\begin{array}{l}\text { Clinical event } \\
\text { prediction: Current } \\
\text { pain level, 3-month } \\
\text { post-op predicted pain } \\
\text { level }\end{array}$ & $\begin{array}{l}\text { Multiple } \\
\text { Regression }\end{array}$ \\
\hline STaRT Back Tool & $\begin{array}{l}\text { Arthritis Care \& Research (2008) } \\
\text { [60], The Journal of Arthroplasty } \\
\text { (2019) [61], Spine (2002) [53] }\end{array}$ & $\begin{array}{c}\text { Prospective Cohort } \\
\text { and Retrospective } \\
\text { Review } \\
1641 \text { patients } \\
\end{array}$ & & Symptoms & $\begin{array}{l}\text { Activity, Pain level, } \\
\text { Mental state }\end{array}$ & $\begin{array}{l}\text { Clinical event } \\
\text { prediction: Chronic } \\
\text { pain risk level }\end{array}$ & $\begin{array}{c}\text { Forward Stepwise } \\
\text { Binary Logistic } \\
\text { Regression } \\
\text { Analysis }\end{array}$ \\
\hline $\begin{array}{l}\text { Estimated Blood } \\
\text { Loss Calculator }\end{array}$ & The Spine Journal (2020) [62] & $\begin{array}{l}\text { Retrospective Cohort } \\
1281 \text { patients }\end{array}$ & BMI & $\begin{array}{c}\text { Pedicle Screws (T11-S1), Pelvic Screws, } \\
\text { Laminectomy, Laminectomy Levels, } \\
\text { Discectomies, ALIF Interbody Fusions, } \\
\text { XLIF/OLIF Interbody Fusions, } \\
\text { TLIF/PLIF Interbody Fusions, Schwab } \\
\text { Osteotomies, TXA Use, Surgery } \\
\text { duration }\end{array}$ & & $\begin{array}{l}\text { Clinical event } \\
\text { prediction: Blood loss } \\
(\mathrm{mL})\end{array}$ & $\begin{array}{l}\text { Univariate Linear } \\
\text { Regressions, } \\
\text { Multivariate } \\
\text { Analysis }\end{array}$ \\
\hline
\end{tabular}


Table 4. Cont.

\begin{tabular}{|c|c|c|c|c|c|c|c|}
\hline Tool & $\begin{array}{l}\text { Journal and (Year) of } \\
\text { Publication [Ref] }\end{array}$ & $\begin{array}{l}\text { Tool Development } \\
\text { Study Type and Size }\end{array}$ & $\begin{array}{c}\text { Demographic } \\
\text { Input }\end{array}$ & Clinical Input & $\begin{array}{l}\text { Patient Reported } \\
\text { Input }\end{array}$ & Tool Output & $\begin{array}{c}\text { Statistical } \\
\text { Methods Used in } \\
\text { Tool } \\
\text { Development }\end{array}$ \\
\hline ShockNurd & $\begin{array}{l}\text { Clinical Orthopaedics and Related } \\
\text { Research (2016) [63] }\end{array}$ & $\begin{array}{l}\text { Retrospective Review } \\
\quad 382 \text { patients }\end{array}$ & Sex & $\begin{array}{l}\text { Tibial nail }>4 \text { weeks ago, Percentage } \\
\text { Cortical Contact, Open Fracture, } \\
\text { Compartment Syndrome, Soft Tissue } \\
\text { Flap Required, Chronic Condition } \\
\text { (HIV/HEP C/Diabetes), ASA } \\
\text { Classification, Low Energy Injury, } \\
\text { Spiral Fracture Pattern }\end{array}$ & & $\begin{array}{l}\text { Clinical event } \\
\text { prediction: NURD } \\
\text { Score, Non-Union } \\
\text { Percentage, } \\
\text { Confidence Range }\end{array}$ & $\begin{array}{l}\text { Bivariate and } \\
\text { Multivariate } \\
\text { Regression, } \\
\text { Stepwise } \\
\text { Modeling }\end{array}$ \\
\hline $\begin{array}{l}\text { Neuro Risk Opioid } \\
\text { Use Calculator }\end{array}$ & Spine (2018) [64] & $\begin{array}{l}\text { Retrospective Cohort } \\
26,553 \text { patients }\end{array}$ & Age, Gender & $\begin{array}{l}\text { Cervical or Lumbar Spine, Operation } \\
\text { type, Diabetes, Depression/Anxiety, } \\
\text { Osteoporosis, Fibromyalgia, Morbid } \\
\text { Obesity, Lower Back Pain, Motor } \\
\text { Deficits (plegia), Bowel/Bladder } \\
\text { dysfunction, Substance abuse, } \\
\text { Preoperative Opioid User (3 Months } \\
\text { Prior to Surgery), }\end{array}$ & & $\begin{array}{l}\text { Clinical event } \\
\text { prediction: Narcotics } \\
\text { use at } 12 \text {-month } \\
\text { postop }\end{array}$ & $\begin{array}{l}\text { Multiple Logistic } \\
\text { Regression }\end{array}$ \\
\hline $\begin{array}{l}\text { Opioid Calculator } \\
\text { for Hand Surgery }\end{array}$ & $\begin{array}{l}\text { The Journal of Hand Surgery (2019) } \\
\text { [65] }\end{array}$ & $\begin{array}{l}\text { Prospective Cohort } \\
526 \text { patients }\end{array}$ & Age & $\begin{array}{l}\text { Can take Naproxen post-op, Can take } \\
\text { Acetaminophen post-op, Currently } \\
\text { taking Narcotics, Planned use of } \\
\text { regional anesthesia, Procedure involves } \\
\text { bone/ligament, Anticipated Surgical } \\
\text { Time }\end{array}$ & & $\begin{array}{l}\text { Clinical event } \\
\text { prediction: Number of } \\
\text { pills to prescribe }\end{array}$ & $\begin{array}{l}\text { Bivariate Analysis } \\
\text { and Multiple } \\
\text { Logistic } \\
\text { Regressions }\end{array}$ \\
\hline $\begin{array}{l}\text { Discharge to } \\
\text { Rehabilitation and } \\
\text { LOS Calculator }\end{array}$ & The Spine Journal (2020) [66] & $\begin{array}{l}\text { Retrospective Cohort } \\
257 \text { patients }\end{array}$ & $\begin{array}{l}\text { Age, BMI, } \\
\text { Insurance }\end{array}$ & $\begin{array}{l}\text { Diabetic, Type of spine surgery, } \\
\text { Procedure time, Elective vs. Emergent }\end{array}$ & & $\begin{array}{l}\text { Processes prediction: } \\
\text { Risk of discharge to } \\
\text { rehab, Length of stay }\end{array}$ & $\begin{array}{c}\text { Univariable And } \\
\text { Multivariable } \\
\text { Analyses }\end{array}$ \\
\hline $\begin{array}{c}\text { RAPT (Risk } \\
\text { Assessment and } \\
\text { Prediction Tool) }\end{array}$ & $\begin{array}{c}\text { The Journal of Arthroplasty (2019) } \\
\text { [67], (2020) [68], (2019) [61], } \\
\text { Clinical Orthopaedics and Related } \\
\text { Research (2015) [69] }\end{array}$ & $\begin{array}{l}\text { Prospective Cohort } \\
3213 \text { patients }\end{array}$ & Age, Sex & & $\begin{array}{c}\text { Functional } \\
\text { Abilities, Social } \\
\text { Support }\end{array}$ & $\begin{array}{l}\text { Processes prediction: } \\
\text { Discharge } \\
\text { requirements, Length } \\
\text { of stay }\end{array}$ & $\begin{array}{l}\text { Binary Logistic } \\
\text { Regression }\end{array}$ \\
\hline
\end{tabular}

Abbreviations: ALIF-Anterior Lumbar Interbody Fusion, AMTS—Abbreviated Mental Test Score, BMD—Bone Mineral Density, BMP—Bone Morphogenetic Protein, CEPOD—Confidential Enquiry into Peri-Operative Deaths, CHF-Congestive Heart Failure, CKD—Chronic Kidney Disease, COPD—Chronic Obstructive Pulmonary Disease, CVA-Cerebrovascular Accident, ECG-Echocardiogram, ECOG-Eastern Cooperative Oncology Group, FEV1-Forced Expiratory Volume, GCS-Glasgow Coma Scale, PLIF-Posterior Lumbar Interbody Fusion, SLE-Systemic Lupus Erythematosus, TIA-Transient Ischemic Attacks, TXA-Tranexamic Acid, XLIF-Extreme Lateral Interbody Fusion, OLIF-Oblique Lumbar Interbody Fusion, TLIF-Transforaminal Lumbar Interbody Fusion. 
We found that the frequency of orthopedic web-based tool publications has increased over time, with $7 \%$ of articles being published before the year 2000, 17\% between 2000 and 2010, and the remaining $76 \%$ between 2010 and 2020 (Figure 1). Tool development was more often informed by exclusively retrospective studies $(n=17,55 \%)$ than by exclusively prospective studies $(n=12,39 \%)$, with only two tools using both retrospective and prospective data sources $(n=2,6 \%)$. Additionally, the sizes of the studies used in tool development varied greatly, with a median study size of 2216 patients, an average study size of 241,644 patients, and a range of 257-4,726,046 patients (Figure 2). While nearly all tools were used clinical inputs $(n=30,97 \%)$ and demographic inputs $(n=29,94 \%)$, a minority used patient-reported inputs $(n=12,39 \%)$. Age was the most commonly used demographic input $(n=25,81 \%)$, with Sex/Gender as the second-most common $(n=16,52 \%)$. Statistical methods used for the creation of tools varied, with most involving logistic regression or multivariate analysis (Table 4 ). Categorization of the tools by output yielded the following breakdown: fracture prediction $(n=7$, $23 \%)$, mortality prediction $(n=9,29 \%)$, clinical event prediction $(n=18,58 \%)$, and processes prediction $(n=3,10 \%)$ (Figure 3$)$. Finally, we found that most tools could be further categorized into orthopedic categories, such as fractures $(n=8,26 \%)$, spine $(n=8,26 \%)$, total joint arthroplasty $(n=5,16 \%)$, oncology $(n=3,10 \%)$, general $(n=5,16 \%)$, and miscellaneous $(n=3,10 \%)$ (Figure 4$)$.

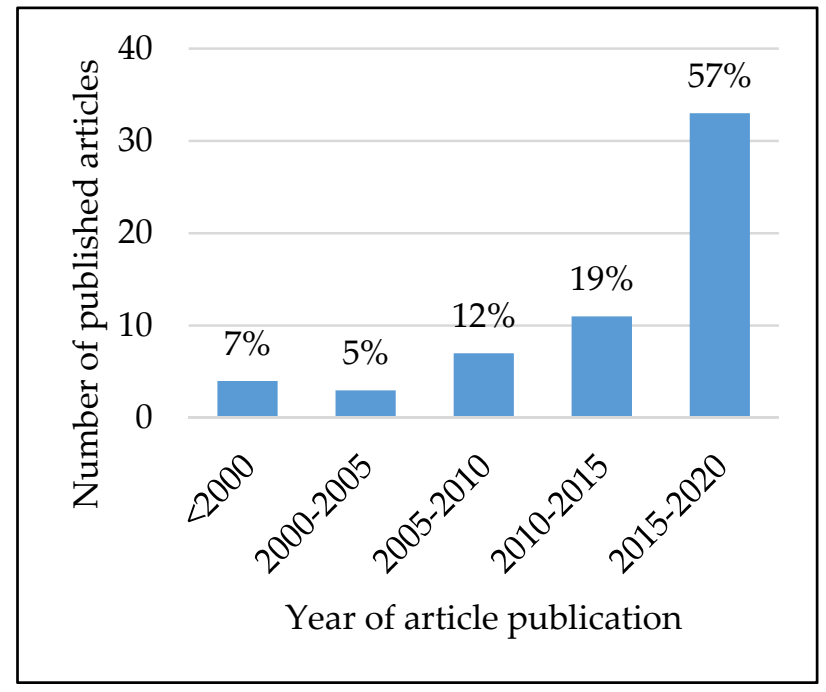

Figure 1. Article publication over time for web-based orthopedic predictive tools.

\begin{tabular}{|c|c|c|}
\hline \multicolumn{2}{|l|}{ Number of Patients } & \multirow[b]{2}{*}{$\mathrm{N}=4$} \\
\hline $0-499$ & $13 \%$ & \\
\hline $500-999$ & $3 \%$ & $\mathrm{~N}=1$ \\
\hline $1000-9999$ & $52 \%$ & $N=16$ \\
\hline $10,000-99,999$ & $13 \%$ & $\mathrm{~N}=4$ \\
\hline $100,000-999,999$ & $13 \%$ & $\mathrm{~N}=4$ \\
\hline $1,000,000+$ & $6 \%$ & $\mathrm{~N}=2$ \\
\hline$\%$ of Total Perc.. & $\begin{array}{l}\% \text { of Total Percentage of Tools } \\
\text { down by Number of Patients. }\end{array}$ & $\begin{array}{l}\text { broken } \\
\text { Color shows }\end{array}$ \\
\hline $52 \%$ & $\begin{array}{l}\% \text { of Total Percentage of Tools. } \\
\text { are based on each column of th }\end{array}$ & $\begin{array}{l}\text { Percents } \\
\text { e table }\end{array}$ \\
\hline
\end{tabular}

Figure 2. Study size used in tool creation. 


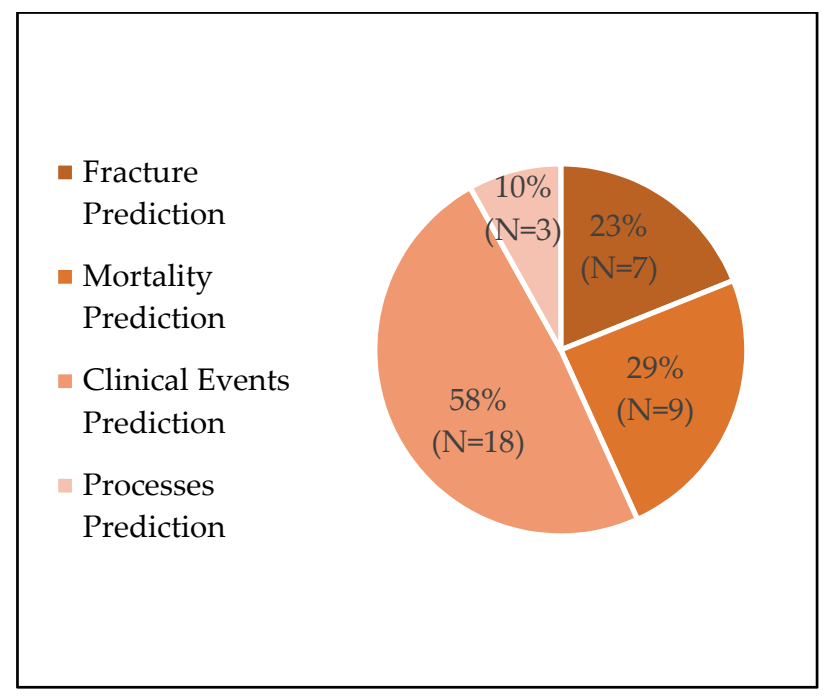

Figure 3. Tool breakdown by output.

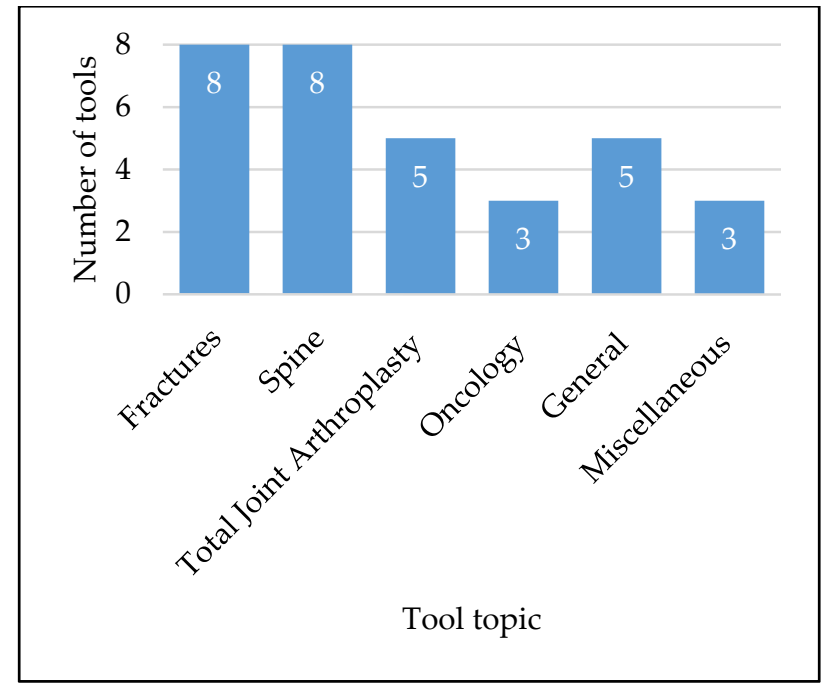

Figure 4. Tool breakdown by orthopedic category.

\section{Discussion}

In this scoping review, we systematically identified 31 web-based tools designed to provide personalized prediction in various of orthopedic settings. Overall, these tools provide orthopedic surgeons with information supporting the outcome prediction of fractures, mortality, other clinical events, such as surgical complications, and miscellaneous clinical processes, such as length of stay. This information applies to settings in different orthopedic subspecialties, in various points of the clinical pathway including pre- and post-operative surgical planning, and for shared decision-making with patients in discussions of elective surgeries.

\subsection{Limitations}

This work should be considered in light of some limitations. Firstly, many identified papers had tools that were inaccessible or had broken URLs. While this may indicate that a given tool was discontinued for technical reasons, it is challenging to ascertain whether there were modifications made for a newer version or alternative use. As tools continue to develop, iterative processes with well-documented updates and modifications will need to be utilized to better implement these tools in clinical practice. Secondly, papers presented varying levels of detail regarding the tool development 
process which limited standardization of specific parameters. Future work should aim to increase transparency in the tool development and validation processes so that tool comparisons are uniform and systematic. Thirdly, only one database was used to search for these tools. While this raises the possibility of missed tools, the two searches conducted for this study were broad and anticipated the capture of the vast majority of these web-based orthopedic solutions. The selected electronic database (PubMed) is also the most relevant to clinical orthopedic practice. Finally, there may be more tools in development that are currently being validated at various institutions and remain unpublished at the time of this review. While this is largely unavoidable in a fast-paced field, further work may target the grey literature and other types of electronic databases or search engines to capture such tools.

It is difficult to quantify the current use of web-based orthopedic predictive tools in practice. This scoping review demonstrates a rapid increase in the frequency of tool-related publications over the last two decades, perhaps reflecting growing interest in web-based orthopedic predictive tools. Although specialized commercial software packages may overshadow the use of web-based tools in areas such as pre-operative planning, web-based tools offer additional and unique functions, such as patient and surgical outcome predictions. In this way, web-based tools serve as potential complements to already established software packages.

Identified tools consistently rely on more established statistical methods for their development, such as multivariate analysis and logistic regression. There were only two tools that demonstrate the use of machine-learning algorithms in their development, both of which were released within the past 5 years. Artificial intelligence and machine learning have the ability to process large amounts of data in different forms, including actively and passively generated data from patients. It is likely that, as this technology evolves, future web-based medical tools will make increasing use of advanced predictive analytics and provide greater opportunities for more personalized patient care.

\subsection{Future Work}

The profile of tools identified in this study indicate two major areas for potential improvement. First, fewer than half of identified tools utilize patient-reported inputs. This may be related to the reliability of obtaining information from patients, as these data require active procurement in prospective cohorts or may not be consistently documented for retrospective cohorts. Despite these barriers, patient reported measures are important components of patient outcomes and ensure physician focus on subjective metrics that patients care about, such as perceived pain. As patient-reported outcome measures are increasingly used in both the field of orthopedics and across medical specialties, greater incorporation of patient-reported data into these tools presents an area of potential improvement. Second, tools identified in this study fall into just a few orthopedic categories, such as fractures, total joint arthroplasty, spine, and oncology. These categories may reflect a lack of tools for other orthopedic subspecialties, such as orthopedic sports injuries. This lack may be related to the heterogeneity of injuries in such fields or insufficient access to large existing patient databases for tool development. Continued expansion in the development of tools across orthopedic subspecialties would afford a wider breadth of resources to orthopedic surgeons to better clinical outcomes for patients and should be a focus of improvement.

\section{Conclusions}

The increasing number of web-based orthopedic tools is an opportunity for orthopedic surgeons to better predict outcomes and increase understanding of expectations with patients. The aim of this scoping review was to identify the current list of web-based orthopedic tools, as well as clearly outline their utility and validation. We provide orthopedic surgeons a repository of current and publicly available web-based tools which includes all the necessary information to determine what tools may apply to their practice. Areas for continued development of web-based tools are vast, with opportunities in both tool design and application. 
Author Contributions: Conceptualization, E.S.; Methodology, E.S., P.C., L.M., and A.C.; Formal Analysis, E.S., P.C., A.C., L.M., E.L., and P.J.; Data Curation, L.M., P.C., and A.C.; Writing-Original Draft Preparation, P.C.; Writing-Review and Editing, E.S., P.C., A.C., L.M., E.L., and P.J.; Supervision, E.S. and P.J. All authors have read and agreed to the published version of the manuscript.

Funding: This research received no external funding.

Conflicts of Interest: The authors declare no conflict of interest.

\section{Appendix A}

Table A1. High Impact Orthopedic Journals Used in Secondary Search.

\begin{tabular}{|c|}
\hline The Journal of Bone and Joint Surgery, American Volume \\
\hline The Bone and Joint Journal \\
\hline Clinical Orthopaedics and Related Research \\
\hline The Journal of the American Academy of Orthopaedic Surgeons \\
\hline Journal of Orthopaedic Research: Official Publication of the Orthopaedic Research Society \\
\hline Journal of Orthopaedics and Traumatology: Official Journal of the Italian Society of Orthopaedics and Traumatology \\
\hline Injury \\
\hline The Journal of Arthroplasty \\
\hline The American Journal of Sports Medicine \\
\hline $\begin{array}{l}\text { Arthroscopy: The Journal of Arthroscopy \& Related Surgery: Official Publication of the Arthroscopy Association of North } \\
\text { America and the International Arthroscopy Association }\end{array}$ \\
\hline The Journal of Hand Surgery \\
\hline Journal of Shoulder and Elbow Surgery \\
\hline Foot $\mathcal{E}$ Ankle Orthopaedics \\
\hline Spine \\
\hline $\begin{array}{c}\text { European Spine Journal: Official Publication of the European Spine Society, the European Spinal Deformity Society, and } \\
\text { the European Section of the Cervical Spine Research Society }\end{array}$ \\
\hline The Spine Journal: Official Journal of the North American Spine Society \\
\hline Journal of Neurosurgery. Spine \\
\hline Joint Bone Spine \\
\hline Journal of Pediatric Orthopedics \\
\hline
\end{tabular}




\section{Appendix B}

Initial PubMeb search:

(((((((calculators[Text Word]) OR (calculator[Text Word])) OR (predictive scores[Text Word])) OR (web based predictive score[ Text Word])) OR (web based calculator[Text Word])) OR (prediction tool[Text Word])) OR (risk calculator[Text Word])) AND ((((c((((orthopaedics[Text Word]) OR (orthopedics[Text Word])) OR (fracture[Text Word])) OR (fractures[Text Word])) OR (break[Text Word])) OR (breaks[Text Word])) OR (hip[Text Word])) OR (hips[Text Word])) OR (orthopedic surgery[Text Word])) OR (orthopaedicsurgery[Text Word]))

Secondary PubMed search:

((()((()((()(((("The Journal of bone and joint surgery. American volume"[Journal]) OR ("The Journal of bone a nd joint surgery. British volume"[Journal])) OR ("Clinical or thopaedics and related research"[Journal])) OR ("The Journal of the American Academy of Or thopaedic Surgeons"[Journal])) OR ("Journal of or thopaedic research: official publication of the Or thopaedic Research Society"[Journal])) OR ("Journal of or thopaedicsand traumatology : official journal of the Italian Society of Or thopaedics and Traumatology"[Journal])) OR ("Inj ury"[Journal])) OR ("The Journal of arthroplasty"[Journal])) OR ("The Americanjournal of sports medicine"[Journal])) OR ("Arthroscopy: thejournal of arthroscopic \& related surgery : official publication of the Arthroscopy Association of Nor th America and the International Arthroscopy Association"[Journal])) OR ("The Journalof hand surgery"[Journal])) OR ("Journal of Shoulder and Elbow Surgery"[Journal])) OR ("Foot \& Ankle Or thopaedics"[Journal])) OR ("Spine"[Journal])) OR ("European spinejournal : official publication of the European Spine Society, the European Spinal Deformity Society, and the European Section of the Cervical Spine Research Society"[Journal])) OR ("The spine journal : official journal of the Nor th American Spine Society"[Journal])) OR ("Journal of neurosurgery. Spine"[Journal])) OR ("Joint bone spine"[Journal])) OR ("Journal of pediatric or thopedics"[Journal])) AND ((((((((w eb [Text Word]) AND (tool[Text Word])) OR (w eb-based[Text Word]))) OR (w eb [Text Word])) AND(predictive[Text Word])) OR (predictive tool[Text Word])) OR (calculator[Text Word]))
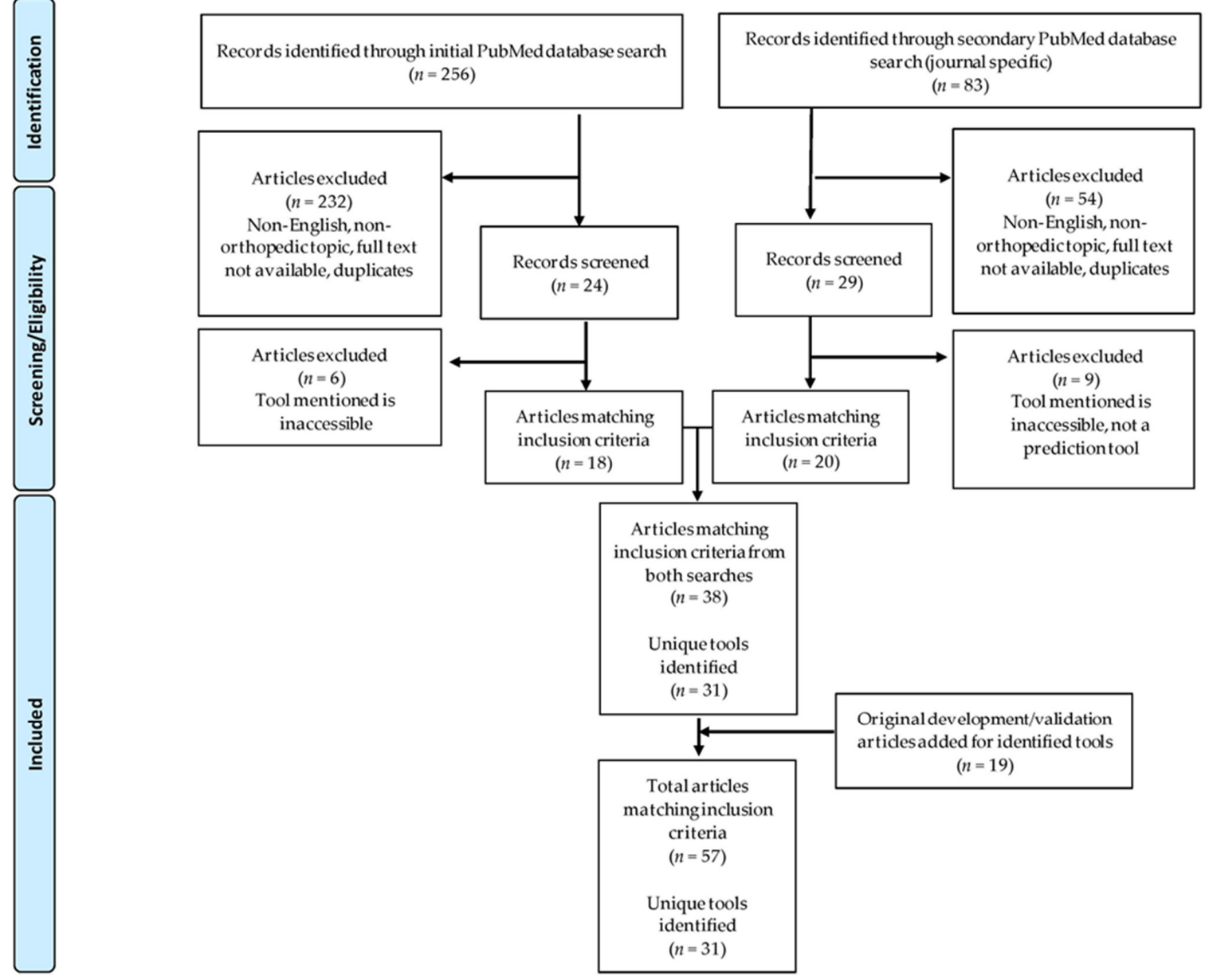

Figure A1. Search Strategy and Identification of Sources. 


\section{Appendix C}

Table A2. Tool URLs.

Tool

URL

Additional Notes

90-Day Readmissions Risk Calculato

https://dukeriskcalculators.shinyapps.io/Readmissions/

(accessed on 26 October 2020)

ACS NSOIP Surgical Risk Calculator $\quad$ https://riskcalculator.facs.org/RiskCalculator/

(accessed on 26 October 2020)

Option to email or download

Arthroplasty Size Predictor

https://apps.apple.com/us/app/arthroplasty-size-predictor/id1234761373

iOS application

Back Treatment Outcomes Calculator

http://spinesurgerycalc.dartmouth.edu/calc/

accessed on 26 October 2020)

CAROC (Canadian Risk for Osteoporosis Calculator)

https://osteoporosis.ca/health-care-professionals/tools/caroc

(accessed on 26 October 2020)

https://www.mdcalc.com/charlson-comorbidity-index-cci (accessed on 26 October 2020)

CCI (Charlson Comorbidity Index)

Chondrosarcoma Five-Year Survival Machine Learning Algorithm

https://sorg-apps.shinyapps.io/chondrosarcoma/

(accessed on 26 October 2020)

Discharge to Rehabilitation and LOS Calculato

https://jhuspine1.shinyapps.io/RehabLOS/

(accessed on 26 October 2020)

Edinburgh Wrist Calculator

https://www trauma.co.uk/wristcalc

(accessed on 26 October 2020)

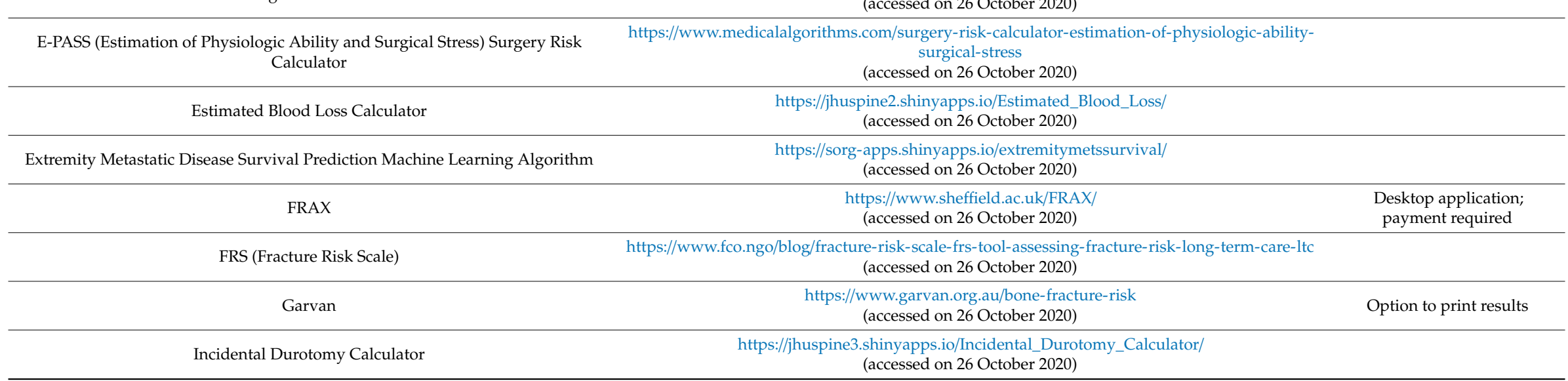


Table A2. Cont.

\begin{tabular}{|c|c|c|}
\hline Tool & URL & Additional Notes \\
\hline LACE+ & $\begin{array}{l}\text { https://www.besler.com/lace-risk-score/ } \\
\text { (accessed on 26 October 2020) }\end{array}$ & \\
\hline Neuro Risk Opioid Use Calculator & $\begin{array}{l}\text { http://neuro-risk.com/opiod-use/ } \\
\text { (accessed on 26 October 2020) }\end{array}$ & \\
\hline NHFS (Nottingham Hip Fracture Score) & $\begin{array}{l}\text { http://www.riskprediction.org.uk/index-nhfs.php } \\
\text { (accessed on 26 October 2020) }\end{array}$ & \\
\hline OaraScore (Outpatient Arthroplasty Risk Assessment Score) & $\begin{array}{l}\text { https://www.djoglobal.com/our-brands/djo-surgical/oara-score } \\
\text { (accessed on 26 October 2020) }\end{array}$ & $\begin{array}{l}\text { Paid subscription required, } \\
\text { option to print results }\end{array}$ \\
\hline Opioid Calculator for Hand Surgery & $\begin{array}{l}\text { https://jscalc.io/calc/9hH05AdFRt4iV6YD } \\
\text { (accessed on 26 October 2020) }\end{array}$ & \\
\hline PathFX & $\begin{array}{l}\text { https://www.pathfx.org/ } \\
\text { (accessed on 26 October 2020) }\end{array}$ & Free registration required \\
\hline P-POSSUM & $\begin{array}{l}\text { http://www.riskprediction.org.uk/index-op.php } \\
\text { (accessed on 26 October 2020) }\end{array}$ & \\
\hline QFracture & $\begin{array}{l}\text { https://qfracture.org/ } \\
\text { (accessed on 26 October 2020) }\end{array}$ & \\
\hline QUALITOUCH Outcome Calculator & $\begin{array}{l}\text { https://outcomecalculator.org/en/ } \\
\text { (accessed on 26 October 2020) }\end{array}$ & \\
\hline RAPT (Risk Assessment and Prediction Tool) & $\begin{array}{l}\text { https://www.lifespan.org/centers-services/total-joint-center-lifespan-orthopedics-institute/take- } \\
\text { rapt-assessment } \\
\text { (accessed on 26 October 2020) }\end{array}$ & \\
\hline ShockNurd & $\begin{array}{c}\text { http://shocknurd.org/ } \\
\text { (accessed on 26 October 2020) }\end{array}$ & Link currently inactive \\
\hline Spinal RAT (Risk Assessment Tool) & $\begin{array}{l}\text { https://apps.apple.com/us/app/risk-assessment-tool-for-spine-surgery-procedures/id1087663216 } \\
\text { (accessed on 26 October 2020) }\end{array}$ & iOS application \\
\hline SpineSage & $\begin{array}{l}\text { https://depts.washington.edu/spinersk/ } \\
\text { (accessed on 26 October 2020) }\end{array}$ & \\
\hline STaRT Back Tool & $\begin{array}{l}\text { https://startback.hfac.keele.ac.uk/training/resources/startback-online/ } \\
\text { (accessed on 26 October 2020) }\end{array}$ & \\
\hline STTGMA (Score for Trauma Triage in Geriatric and Middle Aged Patients) & $\begin{array}{l}\text { https://sttgma.wordpress.com/ } \\
\text { (accessed on } 26 \text { October 2020) }\end{array}$ & $\begin{array}{l}\text { Option to download Excel } \\
\text { formula sheets }\end{array}$ \\
\hline
\end{tabular}




\section{References}

1. Christensen, D.L.; Dickens, J.F.; Freedman, B.; Mauntel, T.; Owens, B.D.; Potter, B.K.; Provencher, M.; Tokish, J.M.; Waterman, B.R.; Antosh, I.; et al. Patient-Reported Outcomes in Orthopaedics. J. Bone Jt. Surg. 2018, 100, 436-442. [CrossRef]

2. Gagnier, J.J. Patient reported outcomes in orthopaedics. J. Orthop. Res. Off. Publ. Orthop. Res. Soc. 2017, 35, 2098-2108. [CrossRef]

3. Sepucha, K.R.; Atlas, S.J.; Chang, Y.; Freiberg, A.; Malchau, H.; Mangla, M.; Rubash, H.; Simmons, L.H.; Cha, T. Informed, Patient-Centered Decisions Associated with Better Health Outcomes in Orthopedics: Prospective Cohort Study. Med. Decis. Mak. Int. J. Soc. Med. Decis. Mak. 2018, 38, 1018-1026. [CrossRef]

4. Bernstein, D.N.; Fear, K.; Mesfin, A.; Hammert, W.C.; Mitten, D.J.; Rubery, P.T.; Baumhauer, J.F. Patient-reported outcomes use during orthopaedic surgery clinic visits improves the patient experience. Musculoskelet. Care 2019, 17, 120-125. [CrossRef]

5. Waheeb, A.; Zywiel, M.G.; Palaganas, M.; Venkataramanan, V.; Davis, A.M. The influence of patient factors on patient-reported outcomes of orthopedic surgery involving implantable devices: A systematic review. Semin. Arthritis Rheum. 2015, 44, 461-471. [CrossRef]

6. Faour, M.; Sultan, A.A.; George, J.; Samuel, L.T.; Curtis, G.L.; Molloy, R.; Higuera, C.A.; Mont, M.A. Arthroscopic irrigation and debridement is associated with favourable short-term outcomes vs. open management: An ACS-NSQIP database analysis. Knee Surg. Sports Traumatol. Arthrosc. 2019, 27, 3304-3310. [CrossRef]

7. McLynn, R.P.; Diaz-Collado, P.J.; Ottesen, T.D.; Ondeck, N.T.; Cui, J.J.; Bovonratwet, P.; Shultz, B.N.; Grauer, J.N. Risk factors and pharmacologic prophylaxis for venous thromboembolism in elective spine surgery. Spine J. 2018, 18, 970-978. [CrossRef]

8. Shillingford, J.N.; Laratta, J.L.; Lombardi, J.M.; Mueller, J.D.; Cerpa, M.; Reddy, H.P.; Saifi, C.; Fischer, C.R.; Lehman, R.A.J. Complications following single-level interbody fusion procedures: An ACS-NSQIP study. J. Spine Surg. (Hong Kong) 2018, 4, 17-27. [CrossRef] [PubMed]

9. Mountain, C.; Redd, R.; O'Leary-Kelly, C.; Giles, K. Electronic medical record in the simulation hospital: Does it improve accuracy in charting vital signs, intake, and output? Comput. Inform. Nurs. 2015, 33, $166-171$. [CrossRef] [PubMed]

10. Bayliss, L.; Jones, L.D. The role of artificial intelligence and machine learning in predicting orthopaedic outcomes. Bone Jt. J. 2019, 101, 1476-1478. [CrossRef] [PubMed]

11. Buch, V.H.; Ahmed, I.; Maruthappu, M. Artificial intelligence in medicine: Current trends and future possibilities. Br. J. Gen. Pract. 2018, 68, 143-144. [CrossRef] [PubMed]

12. Compilation and Analysis of Web-based Orthopedic Predictive Tools: A Scoping Review. Available online: https://osf.io/2e43m (accessed on 11 November 2020).

13. Luokkala, T.; Flinkkilä, T.; Paloneva, J.; Karjalainen, T.V. Comparison of Expert Opinion, Majority Rule, and a Clinical Prediction Rule to Estimate Distal Radius Malalignment. J. Orthop. Trauma. 2018, 32, e97-e101. [CrossRef] [PubMed]

14. Walenkamp, M.M.J.; Mulders, M.A.M.; Van Hilst, J.; Goslings, J.C.; Schep, N.W.L. Prediction of Distal Radius Fracture Redisplacement: A Validation Study. J. Orthop. Trauma. 2018, 32, e92-e96. [CrossRef] [PubMed]

15. Mackenney, P.J.; McQueen, M.M.; Elton, R. Prediction of instability in distal radial fractures. J. Bone Jt. Surg. Am. 2006, 88, 1944-1951.

16. Edwards, B.J. Osteoporosis Risk Calculators. J. Clin. Densitom. Off. J. Int. Soc. Clin. Densitom. 2017, 20, 379-388. [CrossRef] [PubMed]

17. Leslie, W.D.; Lix, L.M. Simplified 10-year absolute fracture risk assessment: A comparison of men and women. J. Clin. Densitom. Off. J. Int. Soc. Clin. Densitom. 2010, 13, 141-146. [CrossRef]

18. Siminoski, K.; Leslie, W.D.; Frame, H.; Hodsman, A.; Josse, R.G.; Khan, A.; Lentle, B.C.; Levesque, J.; Lyons, D.J.; Tarulli, G.; et al. Recommendations for bone mineral density reporting in Canada: A shift to absolute fracture risk assessment. J. Clin. Densitom. Off. J. Int. Soc. Clin. Densitom. 2007, 10, 120-123. [CrossRef]

19. Kanis, J.A.; Harvey, N.C.; Johansson, H.; Odén, A.; McCloskey, E.V.; Leslie, W.D. Overview of Fracture Prediction Tools. J. Clin. Densitom. Off. J. Int. Soc. Clin. Densitom. 2017, 20, 444-450. [CrossRef] 
20. Sharma, A.; Sinha, R.J.; Singh, V.; Garg, G.; Agarwal, S.; Pandey, S. Implications of the Fracture Risk Assessment Algorithm for the assessment and improvement of bone health in patients with prostate cancer: A comprehensive review. Turk. J. Urol. 2019, 45, 245-253. [CrossRef]

21. Negm, A.M.; Ioannidis, G.; Jantzi, M.; Bucek, J.; Giangregorio, L.; Pickard, L.; Hirdes, J.P.; Adachi, J.D.; Richardson, J.; Thabane, L.; et al. Validation of a one year fracture prediction tool for absolute hip fracture risk in long term care residents. BMC Geriatr. 2018, 18, 320. [CrossRef]

22. Ioannidis, G.; Jantzi, M.; Bucek, J.; Adachi, J.D.; Giangregorio, L.; Hirdes, J.; Pickard, L.; Papaioannou, A. Development and validation of the Fracture Risk Scale (FRS) that predicts fracture over a 1-year time period in institutionalised frail older people living in Canada: An electronic record-linked longitudinal cohort study. BMJ Open 2017, 7, e016477. [CrossRef] [PubMed]

23. Nguyen, N.D.; Frost, S.A.; Center, J.R.; Eisman, J.A.; Nguyen, T.V. Development of prognostic nomograms for individualizing 5-year and 10-year fracture risks. Osteoporos. Int. 2008, 19, 1431-1444. [CrossRef] [PubMed]

24. Hippisley-Cox, J.; Coupland, C. Derivation and validation of updated QFracture algorithm to predict risk of osteoporotic fracture in primary care in the United Kingdom: Prospective open cohort study. BMJ 2012, 344, e3427. [CrossRef] [PubMed]

25. Maxwell, M.J.; Moran, C.G.; Moppett, I.K. Development and validation of a preoperative scoring system to predict 30 day mortality in patients undergoing hip fracture surgery. Br. J. Anaesth. 2008, 101, 511-517. [CrossRef] [PubMed]

26. Rushton, P.R.; Reed, M.R.; Pratt, R.K. Independent validation of the Nottingham Hip Fracture Score and identification of regional variation in patient risk within England. Bone Jt. J. 2015, 97, 100-103. [CrossRef]

27. Marufu, T.C.; Mannings, A.; Moppett, I.K. Risk scoring models for predicting peri-operative morbidity and mortality in people with fragility hip fractures: Qualitative systematic review. Injury 2015, 46, 2325-2334. [CrossRef]

28. Veeravagu, A.; Li, A.; Swinney, C.; Tian, L.; Moraff, A.; Azad, T.D.; Cheng, I.; Alamin, T.; Hu, S.S.; Anderson, R.L.; et al. Predicting complication risk in spine surgery: A prospective analysis of a novel risk assessment tool. J. Neurosurg. Spine 2017, 27, 81-91. [CrossRef]

29. Goltz, D.E.; Baumgartner, B.T.; Politzer, C.S.; DiLallo, M.; Bolognesi, M.P.; Seyler, T.M. The American College of Surgeons National Surgical Quality Improvement Program Surgical Risk Calculator Has a Role in Predicting Discharge to Post-Acute Care in Total Joint Arthroplasty. J. Arthroplast. 2018, 33, 25-29. [CrossRef]

30. McCarthy, M.H.; Singh, P.; Nayak, R.; Maslak, J.P.; Jenkins, T.J.; Patel, A.A.; Hsu, W.K. Can the American College of Surgeons Risk Calculator Predict 30-day Complications After Spine Surgery? Spine (Phila. Pa. 1976) 2020, 45, 621-628. [CrossRef]

31. Wingert, N.C.; Gotoff, J.; Parrilla, E.; Gotoff, R.; Hou, L.; Ghanem, E. The ACS NSQIP Risk Calculator Is a Fair Predictor of Acute Periprosthetic Joint Infection. Clin. Orthop. Relat. Res. 2016, 474, 1643-1648. [CrossRef]

32. Edelstein, A.I.; Kwasny, M.J.; Suleiman, L.I.; Khakhkhar, R.H.; Moore, M.A.; Beal, M.D.; Manning, D.W. Can the American College of Surgeons Risk Calculator Predict 30-Day Complications After Knee and Hip Arthroplasty? J. Arthroplast. 2015, 30, 5-10. [CrossRef] [PubMed]

33. Bilimoria, K.Y.; Liu, Y.; Paruch, J.L.; Zhou, L.; Kmiecik, T.E.; Ko, C.Y.; Cohen, M.E. Development and evaluation of the universal ACS NSQIP surgical risk calculator: A decision aid and informed consent tool for patients and surgeons. J. Am. Coll. Surg. 2013, 217, 833. [CrossRef]

34. Jiang, H.X.; Majumdar, S.R.; Dick, D.A.; Moreau, M.; Raso, J.; Otto, D.D.; Johnston, D.W. Development and initial validation of a risk score for predicting in-hospital and 1-year mortality in patients with hip fractures. J. Bone Min. Res. 2005, 20, 494-500. [CrossRef] [PubMed]

35. Haga, Y.; Ikei, S.; Ogawa, M. Estimation of Physiologic Ability and Surgical Stress (E-PASS) as a new prediction scoring system for postoperative morbidity and mortality following elective gastrointestinal surgery. Surg. Today 1999, 29, 219-225. [CrossRef] [PubMed]

36. Haga, Y.; Wada, Y.; Takeuchi, H.; Kimura, O.; Furuya, T.; Sameshima, H.; Ishikawa, M. Estimation of physiologic ability and surgical stress (E-PASS) for a surgical audit in elective digestive surgery. Surgery 2004, 135, 586-594. [CrossRef] [PubMed]

37. Konda, S.R.; Lott, A.; Egol, K.A. Development of a Value-based Algorithm for Inpatient Triage of Elderly Hip Fracture Patients. J. Am. Acad. Orthop. Surg. 2020, 28, e566-e572. [CrossRef] 
38. Konda, S.R.; Seymour, R.; Manoli, A.; Gales, J.; Karunakar, M.A. Development of a Middle-Age and Geriatric Trauma Mortality Risk Score A Tool to Guide Palliative Care Consultations. Bull. Hosp. Jt. Dis. 2016, 74, 298-305.

39. Voskuijl, T.; Hageman, M.; Ring, D. Higher Charlson Comorbidity Index Scores are associated with readmission after orthopaedic surgery. Clin. Orthop. Relat. Res. 2014, 472, 1638-1644. [CrossRef]

40. Caplan, I.F.; Winter, E.; Glauser, G.; Goodrich, S.; McClintock, S.D.; Hume, E.L.; Malhotra, N.R. Composite score for prediction of 30-day orthopedic surgery outcomes. J. Orthop. Res. Off. Publ. Orthop. Res. Soc. 2020, 38, 2189-2196. [CrossRef]

41. Charlson, M.E.; Pompei, P.; Ales, K.L.; MacKenzie, C.R. A new method of classifying prognostic comorbidity in longitudinal studies: Development and validation. J. Chronic. Dis. 1987, 40, 373-383. [CrossRef]

42. Copeland, G.P.; Jones, D.; Walters, M. POSSUM: A scoring system for surgical audit. Br. J. Surg. 1991, 78, 355-360. [CrossRef] [PubMed]

43. Wijesinghe, L.D.; Mahmood, T.; Scott, D.J.; Berridge, D.C.; Kent, P.J.; Kester, R.C. Comparison of POSSUM and the Portsmouth predictor equation for predicting death following vascular surgery. Br. J. Surg. 1998, 85, 209-212. [CrossRef] [PubMed]

44. Van Walraven, C.; Dhalla, I.A.; Bell, C.; Etchells, E.; Stiell, I.G.; Zarnke, K.; Austin, P.C.; Forster, A.J. Derivation and validation of an index to predict early death or unplanned readmission after discharge from hospital to the community. Can. Med. Assoc. J. 2010, 182, 551-557. [CrossRef] [PubMed]

45. Van Walraven, C.; Wong, J.; Forster, A.J. LACE+ index: Extension of a validated index to predict early death or urgent readmission after hospital discharge using administrative data. Open Med. 2012, 6, e80-e90.

46. Thio, Q.C.B.S.; Karhade, A.V.; Ogink, P.T.; Raskin, K.A.; De Amorim Bernstein, K.; Lozano Calderon, S.A.; Schwab, J.H. Can Machine-learning Techniques Be Used for 5-year Survival Prediction of Patients With Chondrosarcoma? Clin. Orthop. Relat. Res. 2018, 476, 2040-2048. [CrossRef]

47. Thio, Q.C.B.S.; Karhade, A.V.; Bindels, B.J.J.; Ogink, P.T.; Bramer, J.A.M.; Ferrone, M.L.; Calderón, S.L.; Raskin, K.A.; Schwab, J.H. Development and Internal Validation of Machine Learning Algorithms for Preoperative Survival Prediction of Extremity Metastatic Disease. Clin. Orthop. Relat. Res. 2020, 478, 322-333. [CrossRef]

48. Forsberg, J.A.; Wedin, R.; Boland, P.J.; Healey, J.H. Can We Estimate Short- and Intermediate-term Survival in Patients Undergoing Surgery for Metastatic Bone Disease? Clin. Orthop. Relat. Res. 2017, 475, 1252-1261. [CrossRef]

49. Piccioli, A.; Spinelli, M.S.; Forsberg, J.A.; Wedin, R.; Healey, J.H.; Ippolito, V.; Daolio, P.A.; Ruggieri, P.; Maccauro, G.; Gasbarrini, A.; et al. How do we estimate survival? External validation of a tool for survival estimation in patients with metastatic bone disease-decision analysis and comparison of three international patient populations. BMC Cancer 2015, 15, 424. [CrossRef]

50. Ehresman, J.; Pennington, Z.; Karhade, A.V.; Huq, S.; Medikonda, R.; Schilling, A.; Feghali, J.; Hersh, A. Incidental durotomy: Predictive risk model and external validation of natural language process identification algorithm. J. Neurosurg. Spine 2020, 1-7. [CrossRef]

51. Lee, M.J.; Cizik, A.M.; Hamilton, D.; Chapman, J.R. Predicting surgical site infection after spine surgery: A validated model using a prospective surgical registry. Spine J. 2014, 14, 2112-2117. [CrossRef]

52. Moulton, H.; Tosteson, T.D.; Zhao, W.; Pearson, L.; Mycek, K.; Scherer, E.; Weinstein, J.N.; Pearson, A.; Abdu, W.; Schwarz, S.; et al. Considering Spine Surgery: A Web-Based Calculator for Communicating Estimates of Personalized Treatment Outcomes. Spine (Phila. Pa. 1976) 2018, 43, 1731-1738. [CrossRef] [PubMed]

53. Birkmeyer, N.J.O.; Weinstein, J.N.; Tosteson, A.N.A.; Tosteson, T.D.; Skinner, J.S.; Lurie, J.D.; Deyo, R.; Wennberg, J.E. Design of the Spine Patient outcomes Research Trial (SPORT). Spine (Phila. Pa. 1976) 2002, 27, 1361-1372. [CrossRef] [PubMed]

54. Kim, K.Y.; Feng, J.E.; Anoushiravani, A.A.; Dranoff, E.; Davidovitch, R.I.; Schwarzkopf, R. Rapid Discharge in Total Hip Arthroplasty: Utility of the Outpatient Arthroplasty Risk Assessment Tool in Predicting Same-Day and Next-Day Discharge. J. Arthroplast. 2018, 33, 2412-2416. [CrossRef] [PubMed]

55. Meneghini, R.M.; Ziemba-Davis, M.; Ishmael, M.K.; Kuzma, A.L.; Caccavallo, P. Safe Selection of Outpatient Joint Arthroplasty Patients With Medical Risk Stratification: The "Outpatient Arthroplasty Risk Assessment Score". J. Arthroplast. 2017, 32, 2325-2331. [CrossRef] 
56. Sershon, R.A.; Li, J.; Calkins, T.E.; Courtney, P.M.; Nam, D.; Gerlinger, T.L.; Sporer, S.M.; Levine, B.R. Prospective Validation of a Demographically Based Primary Total Knee Arthroplasty Size Calculator. J. Arthroplast. 2019, 34, 1369-1373. [CrossRef]

57. Sershon, R.A.; Courtney, P.M.; Rosenthal, B.D.; Sporer, S.M.; Levine, B.R. Can Demographic Variables Accurately Predict Component Sizing in Primary Total Knee Arthroplasty? J. Arthroplast. 2017, 32, 3004-3008. [CrossRef]

58. Goltz, D.E.; Ryan, S.P.; Hopkins, T.J.; Howell, C.B.; Attarian, D.E.; Bolognesi, M.P.; Seyler, T.M. A Novel Risk Calculator Predicts 90-Day Readmission Following Total Joint Arthroplasty. J. Bone Jt. Surg. Am. 2019, 101, 547-556. [CrossRef]

59. Stöckli, C.; Theiler, R.; Sidelnikov, E.; Balsiger, M.; Ferrari, S.M.; Buchzig, B.; Uehlinger, K.; Riniker, C.; Bischoff-Ferrari, H.A. Validity of a simple Internet-based outcome-prediction tool in patients with total hip replacement: A pilot study. J. Telemed. Telecare 2014, 20, 117-122. [CrossRef]

60. Hill, J.C.; Dunn, K.M.; Lewis, M.; Mullis, R.; Main, C.J.; Foster, N.E.; Hay, E.M. A primary care back pain screening tool: Identifying patient subgroups for initial treatment. Arthritis Care Res. (Hoboken) 2008, 59, 632-641. [CrossRef]

61. Zeppieri, K.E.; Butera, K.A.; Iams, D.; Parvataneni, H.K.; George, S.Z. The Role of Social Support and Psychological Distress in Predicting Discharge: A Pilot Study for Hip and Knee Arthroplasty Patients. J. Arthroplast. 2019, 34, 2555-2560. [CrossRef]

62. Pennington, Z.; Ehresman, J.; Molina, C.A.; Schilling, A.; Feghali, J.; Huq, S.; Medikonda, R.; Ahmed, A.K.; Cottrill, E.; Lubelski, D.; et al. A novel predictive model of intraoperative blood loss in patients undergoing elective lumbar surgery for degenerative pathologies. Spine J. 2020. [CrossRef] [PubMed]

63. O’Halloran, K.; Coale, M.; Costales, T.; Zerhusen, T., Jr.; Castillo, R.C.; Nascone, J.W.; O’Toole, R.V. Will My Tibial Fracture Heal? Predicting Nonunion at the Time of Definitive Fixation Based on Commonly Available Variables. Clin. Orthop. Relat. Res. 2016, 474, 1385-1395. [CrossRef]

64. Kalakoti, P.; Hendrickson, N.R.; Bedard, N.A.; Pugely, A.J. Opioid Utilization Following Lumbar Arthrodesis: Trends and Factors Associated With Long-term Use. Spine (Phila. Pa. 1976) 2018, 43, 1208-1216. [CrossRef]

65. Jamieson, M.D.; Everhart, J.S.; Lin, J.S.; Jain, S.A.; Awan, H.M.; Goyal, K.S. Reduction of Opioid Use After Upper-Extremity Surgery through a Predictive Pain Calculator and Comprehensive Pain Plan. J. Hand Surg. Am. 2019, 44, 1050-1059.e4. [CrossRef] [PubMed]

66. Lubelski, D.; Ehresman, J.; Feghali, J.; Tanenbaum, J.; Bydon, A.; Theodore, N.; Witham, T.; Sciubba, D.M. Prediction calculator for nonroutine discharge and length of stay after spine surgery. Spine J. 2020, 20, 1154-1158. [CrossRef] [PubMed]

67. Dibra, F.F.; Silverberg, A.J.; Vasilopoulos, T.; Gray, C.F.; Parvataneni, H.K.; Prieto, H.A. Arthroplasty Care Redesign Impacts the Predictive Accuracy of the Risk Assessment and Prediction Tool. J. Arthroplast. 2019, 34, 2549-2554. [CrossRef] [PubMed]

68. Dibra, F.F.; Parvataneni, H.K.; Gray, C.F.; Vasilopoulos, T.; Prieto, H.A. The Risk Assessment and Prediction Tool Accurately Predicts Discharge Destination After Revision Hip and Knee Arthroplasty. J. Arthroplast. 2020. [CrossRef]

69. Hansen, V.J.; Gromov, K.; Lebrun, L.M.; Rubash, H.E.; Malchau, H.; Freiberg, A.A. Does the Risk Assessment and Prediction Tool Predict Discharge Disposition After Joint Replacement? Clin. Orthop. Relat. Res. 2015, 473, 597-601. [CrossRef]

Publisher's Note: MDPI stays neutral with regard to jurisdictional claims in published maps and institutional affiliations.

(C) 2020 by the authors. Licensee MDPI, Basel, Switzerland. This article is an open access article distributed under the terms and conditions of the Creative Commons Attribution (CC BY) license (http://creativecommons.org/licenses/by/4.0/). 